Article

\title{
Mapping Urban Impervious Surfaces by Using Spectral Mixture Analysis and Spectral Indices
}

\author{
Wenliang Li \\ Department of Geography, Environment, and Sustainability, The University of North Carolina at Greensboro, \\ Greensboro, NC 27412, USA; w_li3@uncg.edu
}

Received: 19 November 2019; Accepted: 23 December 2019; Published: 26 December 2019

\begin{abstract}
Impervious surface is the major component of urban areas, and it has been widely considered as the key for assessing the degree of urban sprawl. While the effectiveness of applying spectral mixture analysis (SMA) and spectral indices in mapping urban impervious surface has been proved, most studies have relied either on SMA or spectral indices without considering both. In this study, the SMA and spectral indices were integrated together to map impervious surfaces distributions in both Milwaukee County in the Wisconsin State and Fayette County in the Kentucky State. Specifically, spectral indices were used for identifying major land covers. Two-dimensional feature space plots were generated by calculated spectral indices images for endmember selection and extraction. Linear constrained SMA was finally applied to quantify the fractional impervious surfaces. Research results indicate that the proposed method has achieved a promising accuracy, and better performance was achieved in less developed areas than the developed areas. Moreover, a comparative analysis shows that the proposed method performs better than the conventional method in both the whole study area and the developed areas, and a comparable performance has been achieved in the less developed areas.
\end{abstract}

Keywords: spectral mixture analysis; impervious surface; comparative analysis; spectral index; Landsat

\section{Introduction}

Since the second industrial revolution in the late 19th century, the world has experienced rapid urbanization. The percentage of people who reside in cities raised from $13.3 \%$ in 1900 to $35.6 \%$ in 1970 and $55.3 \%$ in 2018, and it is projected to be over 68\% in 2050 [1]. Associated with rapid urbanization, large numbers of natural lands (e.g., grassland and forest area) were replaced by residential, transportation, industrial, and commercial land, which is majorly composed by impervious surfaces [2-4]. Impervious surface is generally defined as human-made features that water cannot go through (e.g., building rooftop, driveway, and parking areas). As the main part of urban areas, the impervious surface was commonly assumed as an important indicator for evaluating the degree of urban sprawl [5-8]. Therefore, the accurate mapping of impervious surfaces has become an important research topic.

Several methods were proposed and used for mapping impervious surfaces at different scales. Until now, manual interpretation has been considered as the most straightforward and accurate method. However, it is time-consuming and difficult to be conducted in a large geographical region. Since the launch of the first remote sensing satellite, remote sensing data have become widely used in urban sprawl and land use land cover change studies as the large area coverage, regular revisit period, and multiple spectral bands [9]. Since then, conventional classification techniques, such as supervised and unsupervised classifications, have been applied in the estimation of impervious surfaces at different scales. Although the conventional classification approaches are all straightforward, the classification is only conducted at the pixel level. In fact, the spatial extent of a typical land cover is usually smaller 
than the pixel size, and there may be a lot of mixed pixels in an image owing to the variations of land use combinations in urban areas, which result in less accurate estimation results [10]. To address this issue, Ridd [11] proposed the vegetation-impervious surface-soil model (V-I-S) to quantify the components of the urban environment at the sub-pixel scale with the assumption that the land use land cover (LULC) types can be decomposed as the areal abundances of impervious surface, soil, and vegetation. Based on the conceptual V-I-S model, numerous automated methods have been proposed, and they can be grouped into three major types: (1) regression/decision tree (RT/DT), (2) artificial neural networks (ANN), and (3) spectral mixture analysis (SMA) [3,9,12,13].

The DT/RT model is used to construct the relationship between a dependent variable and independent variables by breaking up the data under certain specific rules over and over again, and the ANNs model is a self-adjustment system, which modifies its composition through the learning process to analyze and simulate the input and output data's relationship. They are both considered as machine learning methods and also have been widely used for impervious surface estimation through constructing the empirical relationship with spatial and spectral characteristics offered by remote sensing data [7,14-21]. However, the estimation accuracy of both methods is highly decided by the chosen training data and test data, which is subjective for the model construction and validation [22,23]. Furthermore, some ANNs models, such as the Multi-Layer Perceptron model, require both presence and absence data, and true and false information, which make the data collection rather challenging.

SMA is seen as one powerful approach for the estimation of impervious surfaces in recent years [3,24-26]. It has also been applied in many fields, such as geological mapping [27-29], natural hazard risk assessment [30,31], vegetation mapping [32-35], etc. SMA uses a linear/nonlinear model to explain the relationship between the spectra of a mixed pixel and the spectra of composed land use land covers, and such relationships can help in deriving the areal abundances of each land use/land cover based on the inverse least square's deconvolution model. Until now, the SMA model was widely employed in many studies, especially for mapping the abundances of impervious surfaces [3,12,25,36-44]. Besides, many studies have also been conducted for improving the performance of the SMA model for estimating urban impervious surfaces. Wu and Murray [3] extracted the abundances of low and high albedos separately and combined them to map the impervious surface fractions. Wu [12] has also proposed a normalized SMA to increase the mapping accuracy by lowering the brightness variations among different endmember classes. Zhang et al. [45] developed a prior knowledge-based SMA. In particular, the study area has been segmented into different areas based on the urban intensity, and then unmixing analysis has been applied separately for the segmented areas. Some other proposed popular techniques include derivative spectral unmixing (DSU) [46], segmentation-based SMA [47], spectral weighting techniques [48], spatially adaptive SMA(SASMA) [49], and multiple endmember spectral mixture analysis (MESMA) [50]. Recently, Li and $\mathrm{Wu}$ [51] proposed phenology based temporal mixture analysis (TMA). Instead of applying image reflectance, a vegetation index, Normalized Difference Vegetation Index (NDVI), has been applied, and the phenology profile of endmember classes has been extracted and applied for impervious surface estimation [52-54].

To map impervious surfaces, some spectral indices have also been developed and applied, such as the Normalized Difference Built-up Index (NDBI) [55] and Biophysical Composition Index (BCI) [56]. NDBI is developed for mapping built-up in urban areas through identifying the significantly different spectral profiles between built-up and other LULC types. BCI was introduced by Deng and Wu [56], and instead of identifying only one land cover, BCI can effectively measure the density for several different land covers, such as high albedo, low albedo, soil, and vegetation. Moreover, some other spectral indices have also been proposed for mapping other land features and reducing the confusion with impervious surfaces. For instance, the NDVI is the most commonly used spectral index in mapping vegetation, and it is usually calculated by measuring the differences between red and near-infrared. It is useful in understanding vegetation greenness and has been widely applied to determine the vegetation density on a patch of land. The Ratio Normalized Difference Soil Index (RNDSI) is proposed 
for highlighting soil information in both urban and rural areas using satellite images, it is powerful in separating soil from other land use/land covers (e.g., vegetation and impervious surface), and it could be applied in both urban and nonurban environments [57].

While the effectiveness in applying spectral mixture analysis and spectral indices in quantifying impervious surface has been proven, most studies have relied either on spectral mixture analysis or spectral indices without considering both. In this study, take Milwaukee County in the State of Wisconsin and Fayette County in the Kentucky State as study areas, the urban impervious surfaces mapping were improved by using both spectral mixture analysis and spectral indices. In particular, spectral indices, including NDVI, NDBI, RNDSI, and high and low albedos (from BCI), were applied for identifying major land covers in the selected research regions and further guiding the endmember selection and extraction. Furthermore, a constrained linear SMA was applied for quantifying the fractional impervious surfaces.

\section{Materials and Methods}

\subsection{Study Area}

Milwaukee County in the State of Wisconsin and Fayette County in the Kentucky State were selected in this study to test and verify the proposed method. Milwaukee county is situated in the southeastern part of the Wisconsin State along Lake Michigan with the latitude of $43^{\circ} 2^{\prime} \mathrm{N}$, the longitude of $87^{\circ} 54^{\prime} \mathrm{W}$, and a geographical area of $3079 \mathrm{~km}^{2}$ (Figure 1). The climate is a typical humid continental climate, which is hot in summer and cold and snowy in winter. Fayette County is in the central part of the Kentucky State with the latitude of $37^{\circ} 59^{\prime} \mathrm{N}$ and longitude of $84^{\circ} 28^{\prime} \mathrm{W}$. The total area of Fayette County is $740 \mathrm{~km}^{2}$. The climate is typically mild and moderate with four distinct seasons; summer is warm, and winter is moderately cold. (Figure 1). Both Milwaukee County and Fayette County have experienced rapid urbanization in the past decades, and it is expected to continue in the future. U.S. Census Bureau reports that the population of Milwaukee County and Fayette County has reached 952,085 and 321,959 in 2017, respectively. This rapid urbanization brings a lot of benefits (e.g., economic growth), but also caused several challenging problems, including air pollution, traffic congestion, etc. As the impervious surface is the main part of the urbanized area and has also been widely applied for evaluating the degree of urban sprawl, the accurate mapping of urban impervious surfaces in Milwaukee County and Fayette County becomes more and more important. 


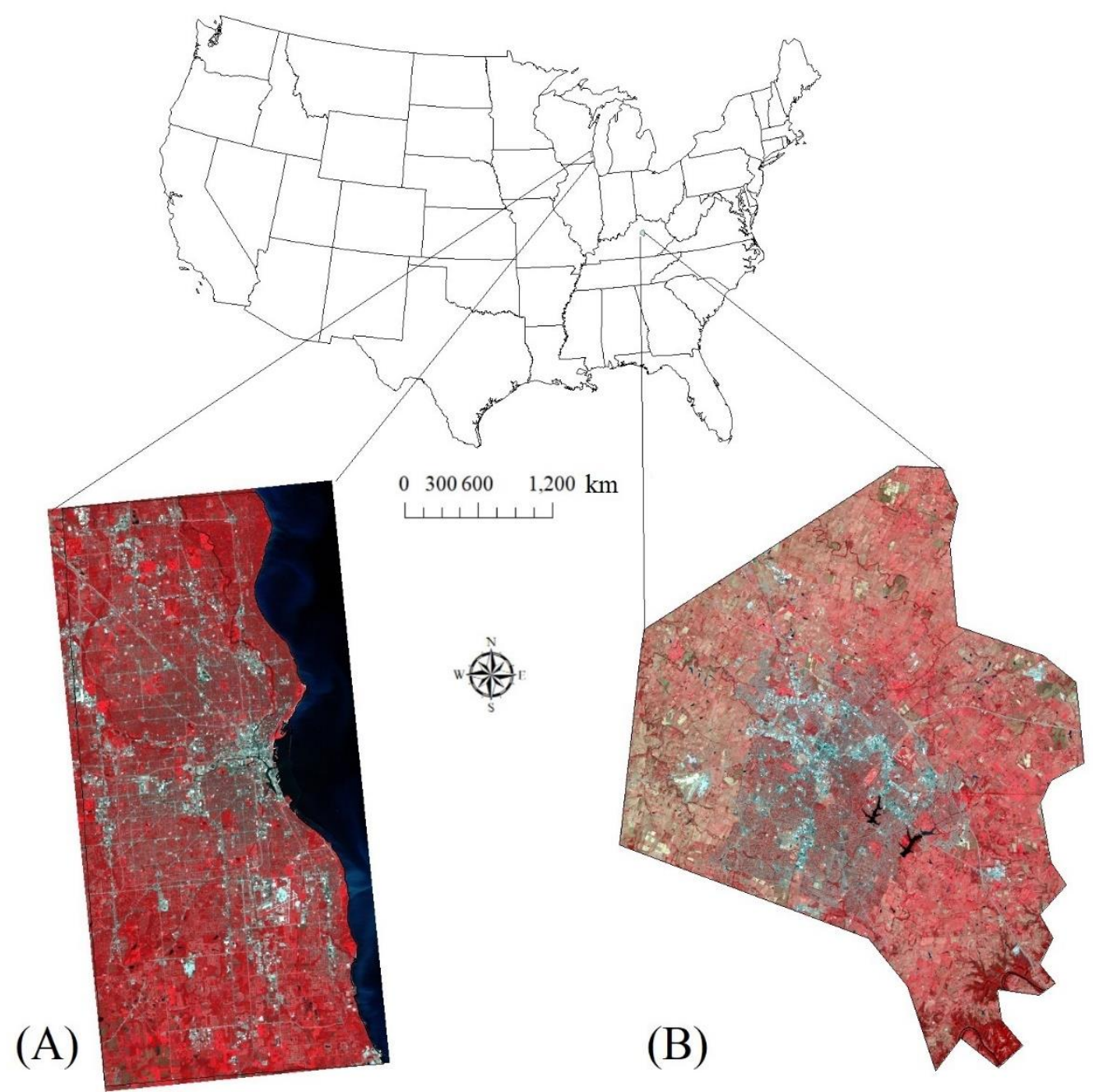

Figure 1. Map of the study areas: (A) Milwaukee County, Wisconsin and (B) Fayette County, Kentucky.

\subsection{Dataset}

To map fractional impervious surfaces in Milwaukee County and Fayette County through the proposed approach, two Landsat Thematic Mapper (TM) images with 6 bands (the thermal band was excluded) acquired on the 12 September 2010 and the 23 September 2010 were applied. The images were collected from the official website of the United States Geological Survey (USGS). The collected images have an appropriate resolution $(30 \mathrm{~m})$ to capture urban features. Image reflectance was calculated based on the digital number of the collected remote sensing images using ERDAS IMAGINE. In addition to the Landsat TM data, I also collected the digital orthophotography images of Milwaukee County on 5 October 2010, with a fine spatial resolution as the references for accuracy assessment. The digital orthophotography images were obtained from the American Geographical Society Library at UW-Milwaukee library. Moreover, the images of Fayette County with fine resolution were collected from Google Earth (recorded in December 2010) and used as references to verify the performance of the proposed method. With the V-I-S model, the urban area was composed of vegetation, impervious surface, and soil. Wu and Murray [3] further clarified that urban impervious surface can be explained by high albedos and low albedos, and the urban environment could be considered as the combination of vegetation, high albedo, low albedo, and soil. Therefore, four endmembers, including vegetation, high albedo, low albedo, and soil, are considered in this study for unmixing analysis. 


\subsection{Methodology}

The proposed method could be carried out in three major steps: major land covers mapping through calculating spectral indices, endmember class selection and extraction, and constrained linear SMA (Figure 2). The detail of the proposed method is included below.

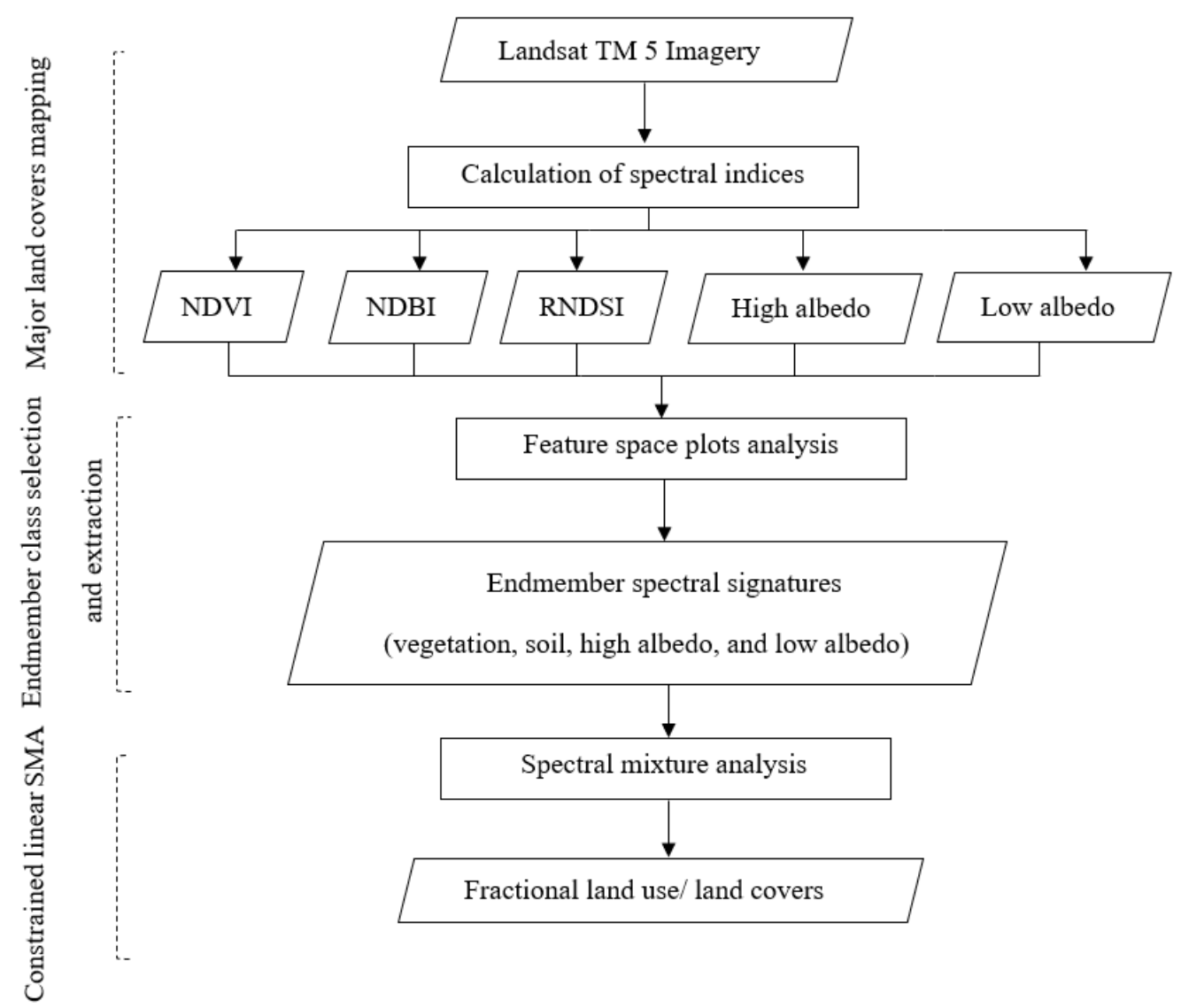

Figure 2. Flowchart of the proposed method.

\subsubsection{Mapping Major Land Covers Using Spectral Indices}

Five spectral indices, NDVI [58], NDBI [55], RNDSI [57], and high and low albedo (from BCI) [56], were employed in this research to map major land use/land covers in the research regions. The generalized expressions of these spectral indices are listed as follows:

$$
\begin{aligned}
& N D V I=\frac{N I R-\text { Red }}{N I R+R e d} \\
& N D B I=\frac{M I R-N I R}{M I R+N I R}
\end{aligned}
$$

where NIR represents the reflectance obtained in the near-infrared band, and Red is the reflectance collected in the red bands. MIR is the reflectance derived in the medium infrared band.

$$
\begin{gathered}
R N D S I=\frac{N N D S I}{N T C I} \\
N N D S I=\frac{N D S I-N D S I_{\text {min }}}{N D S I_{\text {max }}-N D S I_{\text {min }}} \\
N D S I=\frac{\text { Band } 7-\text { Band } 2}{\text { Band } 7+\text { Band } 2}
\end{gathered}
$$




$$
T C I=\frac{T C 1-T C 1_{\text {min }}}{T C 1_{\text {max }}-T C 1_{\text {min }}}
$$

where NDSI represents the normalized difference soil index. NNDSI is the normalized NDSI. NDSI max is the maximum value of the calculated NDSI, and NDSI $I_{\min }$ is the minimum value of the calculated NDSI. Band7 is the seventh band of the collected image, and Band2 is the second band of the obtained image. TC1 is the first component of the image after the tasseled cap transformations, and NTC1 is the normalized TC1. For details, please refer to Deng et al. [57].

$$
\begin{aligned}
& H=\frac{T C 1-T C 1_{\min }}{T C 1_{\max }-T C 1_{\min }} \\
& L=\frac{T C 3-T C 3_{\min }}{T C 3_{\max }-T C 3_{\min }}
\end{aligned}
$$

where $H$ and $L$ are high albedos and low albedo from the BCI. TC1 is the first component, and TC3 is the third component of the TM image after the tasseled cap transformation. TC $1_{\text {max }}$ is the maximum value of the $T C 1$, and $T C 1_{\text {min }}$ is the minimum value of the TC1. TC $3_{\text {max }}$ is the maximum value of the $T C 3$, and $T C 3_{\min }$ is the minimum value of the TC3. For details, please refer to Deng and Wu [56].

\subsubsection{Selecting and Extracting Endmembers}

Selecting the endmembers is the key step for spectral mixture analysis. In general, statistical analysis was applied (e.g., Principal component analysis (PCA) and Minimum noise fraction (MNF)) for helping the endmember selection. In this study, instead of applying PCA or MNF, five spectral indices were used in selecting endmembers. In order to generate feature space plots for guiding the endmember selection and extraction, ERDAS IMAGINE was used in this study. Specifically, all spectral indices images were stacked as one image with five bands using the layer stack function. Furthermore, the feature space plot function was applied to generate all two-dimensional feature space plots, and the vertices of those two-dimensional scatter plots were identified as endmembers. In addition, the selected endmembers were also verified with ground reference data (Figure 3). These endmembers include vegetation, high albedo, low albedo, and soil.
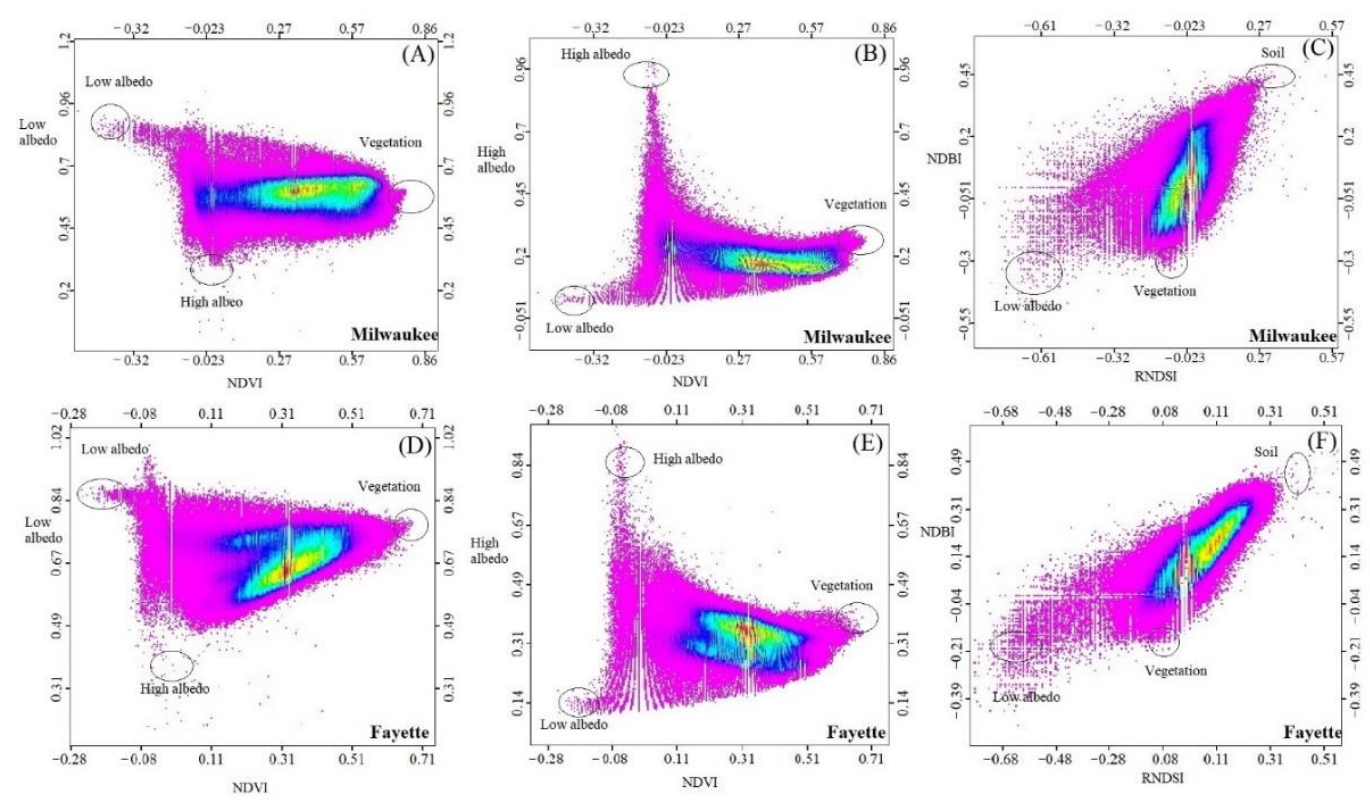

Figure 3. Two-dimensional feature space plots generated from spectral indices for both Milwaukee County: (A) NDVI-Low albedo (B) NDVI-High albedo (C): RNDSI-NDBI and Fayette County: (D) NDVI-Low albedo (E) NDVI-High albedo (F) RNDSI-NDBI. 


\subsubsection{Constrained Linear SMA}

Based on the selected endmembers, a constrained linear SMA was applied. The spectra of the mixed pixels could be linearly explained by the spectra of all endmembers, and the abundances of all endmembers could be extracted accordingly. The constrained linear SMA and corresponding constrain could be expressed as follows.

$$
\begin{gathered}
R_{b}=\sum_{i=1}^{N} R_{i, b} f_{i}+e_{b} \\
\text { Subject to } \sum_{i=1}^{N} f_{i}=1 \text { and } f_{i} \geq 0
\end{gathered}
$$

where $R_{b}$ is the mixed spectral index value at band $b, \mathrm{~N}$ is the total amount of endmember classes (four endmember classes are included in this study), $f_{i}$ is the areal abundances of endmember class $i, R_{i, b}$ is the spectral index value for endmember $i$ at band $b$, and $e_{b}$ is the residual. To evaluate the model performance, the $e_{b}$ and RMS have been calculated and could be expressed as follows,

$$
R M S=\left(\frac{\sum_{b=1}^{M} e_{b}^{2}}{M}\right)^{\frac{1}{2}}
$$

where $M$ is the total amount of bands (5 spectral indices images).

\subsubsection{Conventional SMA}

In order to compare the performance of the proposed method with conventional SMA, the conventional SMA was also implemented in this study. With conventional SMA, the reflectance has been calculated for each image and an MNF has been applied to remove noise (Figure 4). Endmember classes were selected from the first three components of MNF, and the reflectance images were applied for spectral mixture analysis. The same as in the proposed method, four endmembers, including vegetation, high albedo, low albedo, and soil, were included in this study. The constrained linear SMA could be expressed as follows.

$$
R E_{b}=\sum_{i=1}^{N} R E_{i, b} f_{i}+e_{b}
$$

where $R E_{b}$ is the mixed reflectance value at band $b, \mathrm{~N}$ is the total amount of endmember classes (four endmember classes are included in this study), $f_{i}$ is the areal abundances of endmember class $i, R E_{i, b}$ is the reflectance for endmember $i$ at band $b$, and $e_{b}$ is the residual.

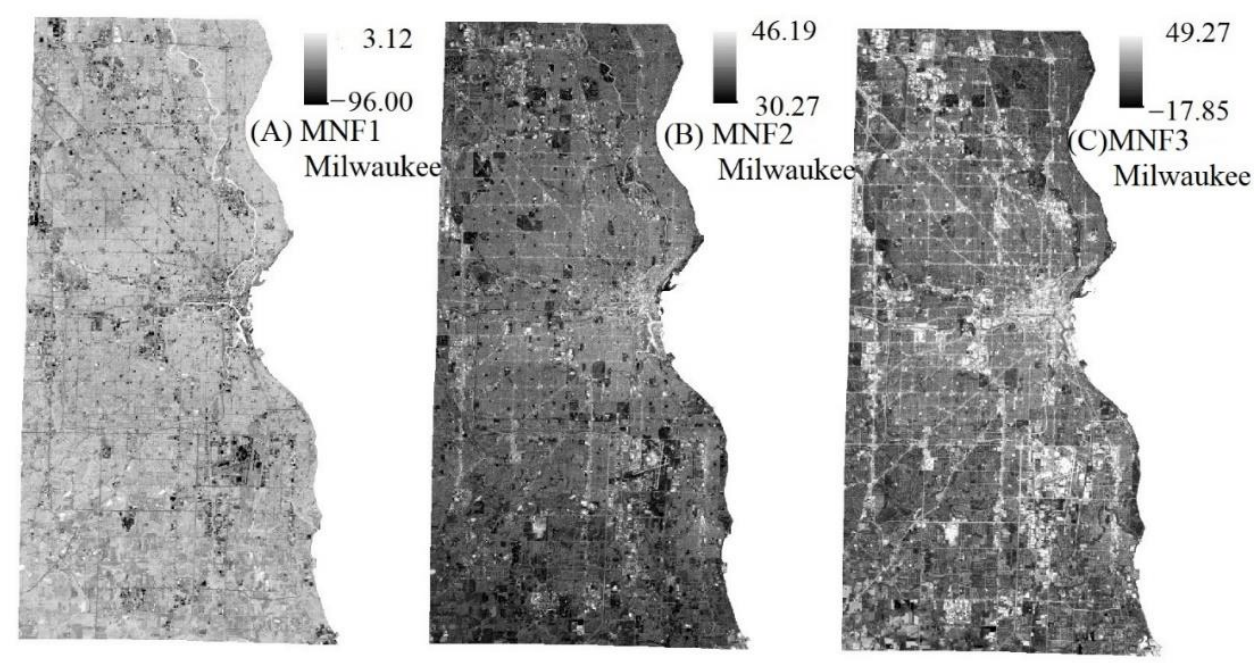

Figure 4. Cont. 

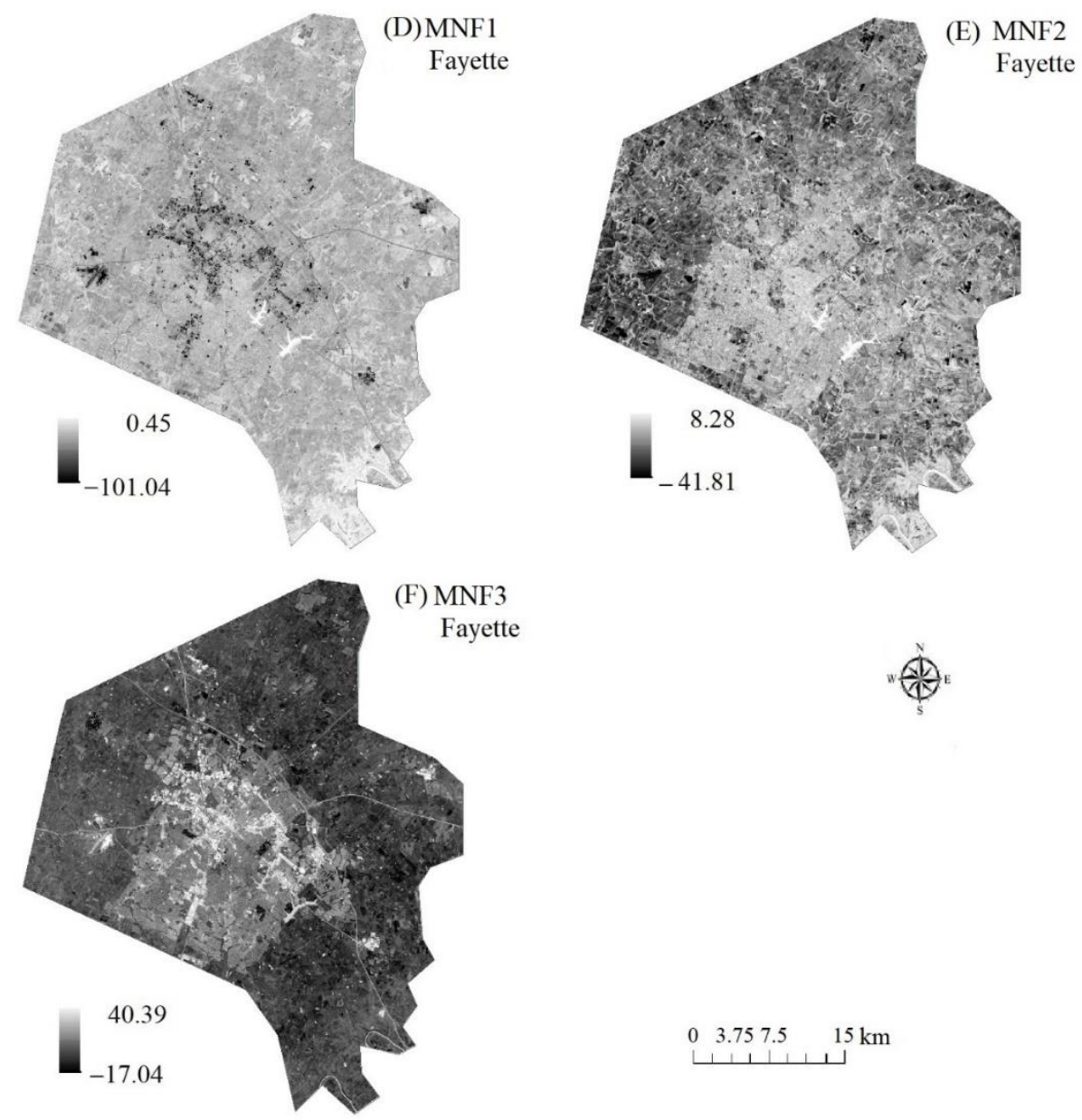

Figure 4. Minimum noise fraction (MNF) images derived for Milwaukee County: (A) MNF1 (B) MNF2 (C) MNF3 and Fayette County: (D) MNF1 (E) MNF2 (F) MNF3.

\subsubsection{Accuracy Assessment}

To assess the model performance of the proposed method and conventional SMA, the digital orthophotography images of Milwaukee County on 5 October 2010 and the Google Earth images of Fayette County (with fine resolution) on December 2010 were collected and used in this study as the reference data. Moreover, two widely used measures, including systematic error (SE, Equation (12)) and mean absolute error (MAE, Equation (13)) were employed to assess the performance of the developed new method $[3,49,51,59,60]$. The $\mathrm{SE}$ is the percentage of estimation error calculated based on the mapped impervious surface and referenced impervious surface; it can reflect the bias and the overall tendency for under- or overestimation. The MAE is the absolute estimation error percentage calculated using the mapped impervious surface and referenced the impervious surface, and it can measure the relative estimation errors of the fractional impervious surface. The details about the two measures, SE and MAE, could be expressed as below,

$$
\begin{gathered}
S E=\frac{\sum_{i=1}^{P}\left(\overline{F_{i}}-F_{i}\right)}{P} \\
M A E=\frac{\sum_{i=1}^{P}\left|F_{i}-\overline{F_{i}}\right|}{P}
\end{gathered}
$$

where $\overline{F_{i}}$ is the simulated abundances of impervious surfaces from the proposed new method at pixel $i$, $F_{i}$ is the referenced abundances of impervious surface at pixel $i$, and $P$ is the total amount of pixels. 


\section{Results}

\subsection{Mapped Major Land Cover Distributions}

The distribution of high and low albedo, soil, built-up, and vegetation of Milwaukee County and Fayette were reported in Figure 5A-J based on the calculation of spectral indices. It illustrates different spatial patterns of LULC in Milwaukee County and Fayette County. In Milwaukee County, the built-ups (composed by both high albedos and low albedos) are majorly distributed in the central eastern part of the Milwaukee County, along Lake Michigan. Vegetations, mainly forests and crops, are situated in the southwestern part of Milwaukee County. In Fayette County, the built-ups are located in the central part of Fayette County as the largest city, Lexington, is situated there. Vegetations, mainly cropland, are distributed in the outlier of the County. In both counties, the soil is mixed together with croplands and some built-ups.
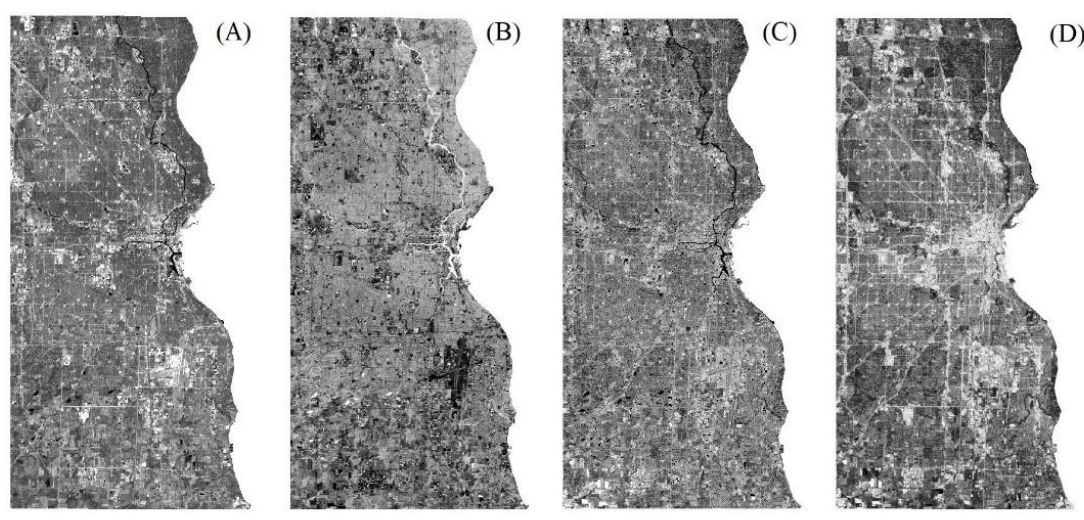

(D)
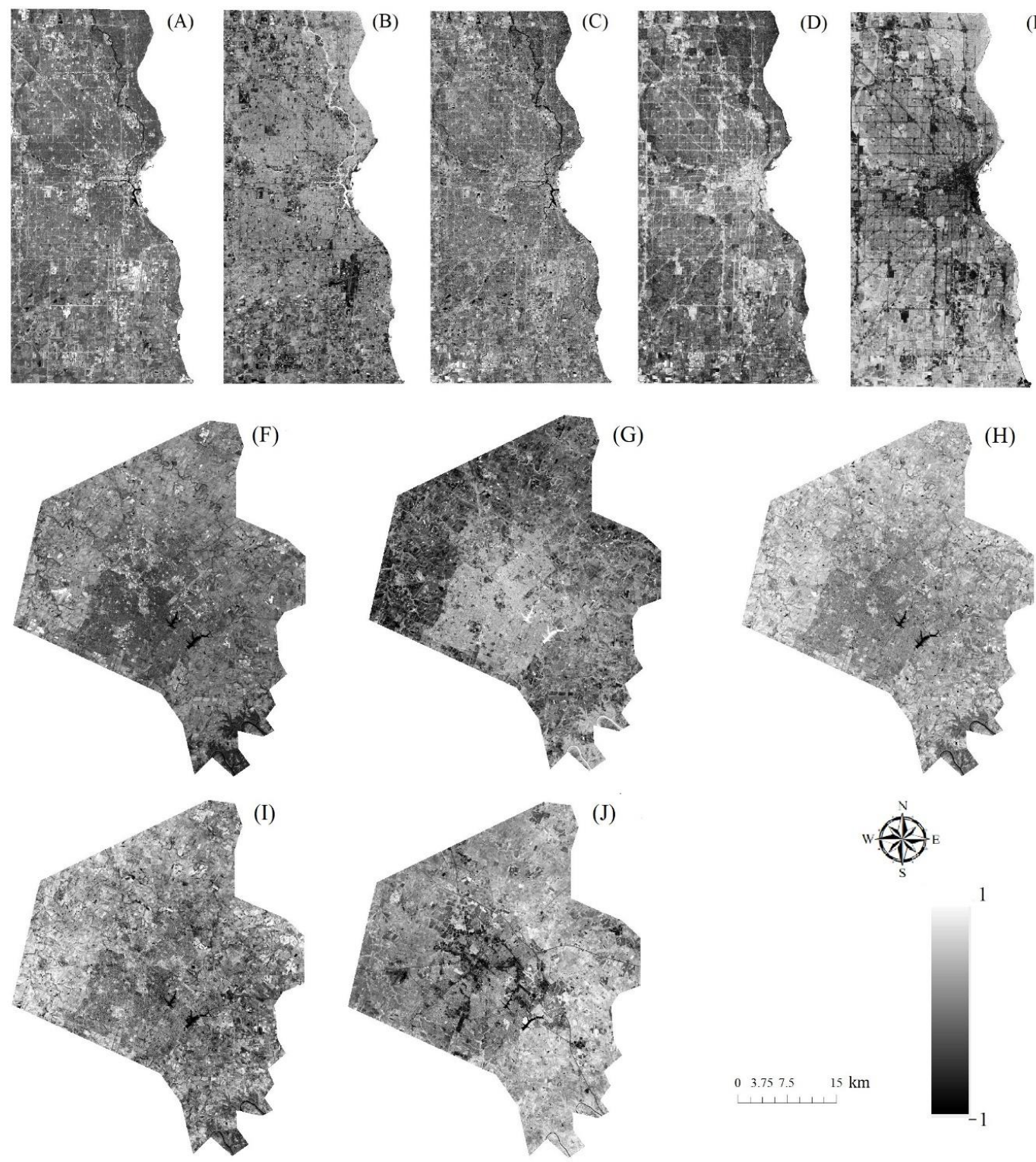

Figure 5. Spectral indices images: (A,F) High albedo, (B,G) Low albedo, $(\mathbf{C}, \mathbf{H})$ Ratio Normalized Difference Soil Index (RNDSI), (D,I) Normalized Difference Built-up Index (NDBI), (E,J) Normalized Difference Vegetation Index (NDVI). 


\subsection{Quantified Impervious Surface Distribtuions}

With the derived spectral indices images, pure endmembers were selected and applied in unmixing analysis to extract four fractional images: high albedo, low albedo, vegetation, and soil. In most studies, the impervious surface was considered as the combination of high albedo and low albedo. Therefore, in this study, the fractional impervious surface map was generated by combining the quantified high albedo and low albedo images (Figure 6). Figure 6A,C illustrate that the spatial distributions of the quantified impervious surface fractions are consistent with the known land use/land cover pattern of Milwaukee County and Fayette County. For Milwaukee County, high abundances of impervious surfaces (AIS $>70 \%$ ) are majorly distributed in the most urbanized and populated areas, such as the City of Milwaukee (the largest city in Milwaukee County). Medium abundances of impervious surfaces (AIS: $30-70 \%$ ) are located in the satellite cities around the City of Milwaukee, such as West Allis, Shorewood, and Glendale, which are commonly recognized as residential communities. Moreover, low abundances of impervious surfaces (AIS $<30 \%$ ) are majorly located in the southern and northern areas of Milwaukee County, which are mostly recognized as vegetation, such as crops, forests, and grasses. For Fayette County, the high fractional impervious surfaces (AIS > 70\%) are mainly located in the largest city and also the center of the County, the City of Lexington. Medium fractional impervious surfaces (AIS: $30-70 \%$ ) are distributed around the City of Lexington, especially the southwestern part of the County, which is majorly covered by houses and apartments. The low fractional impervious surfaces (AIS $<30 \%$ ) are distributed on the outskirts of the county, which are mostly covered by croplands.

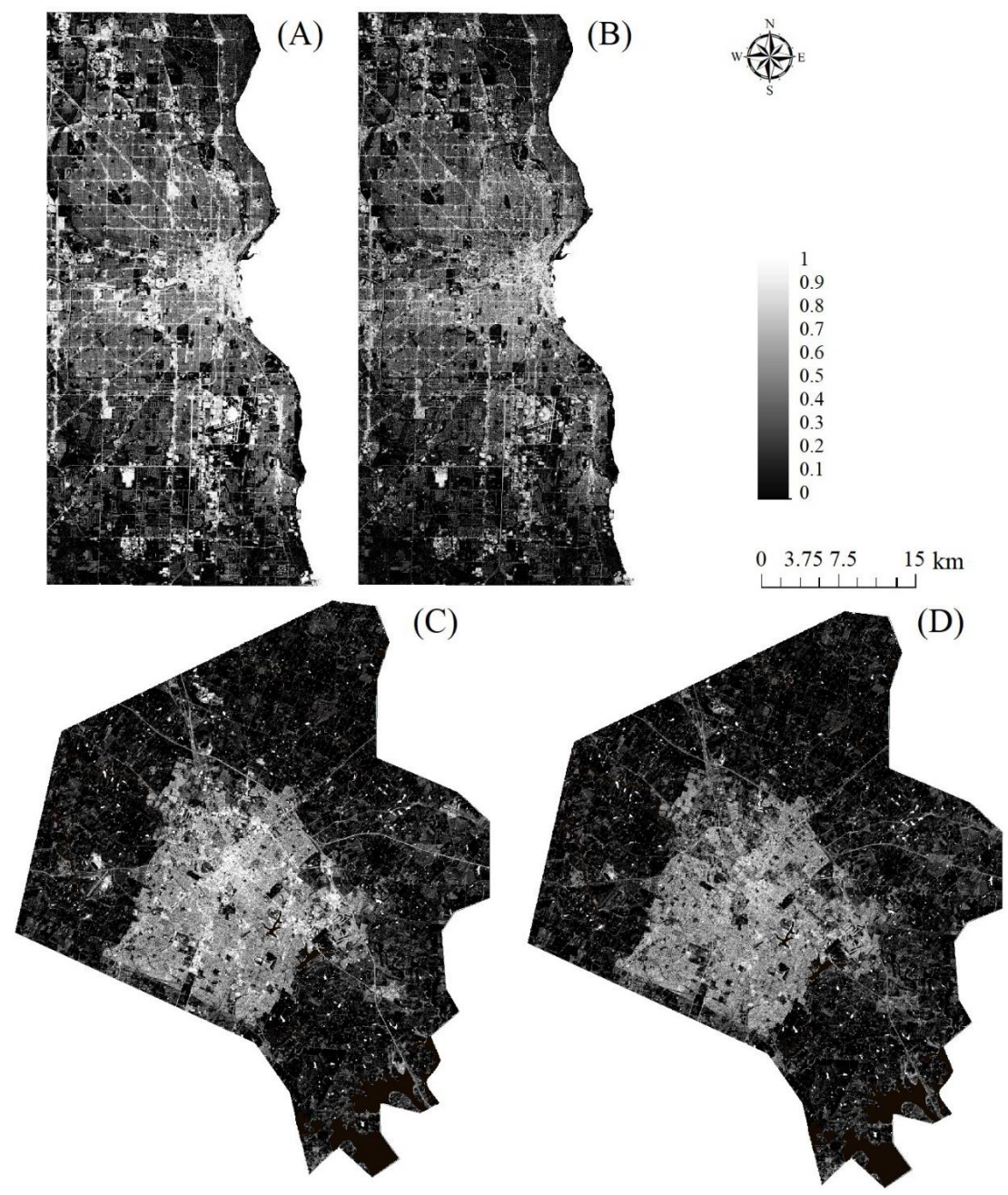

Figure 6. Abundances of impervious surfaces extracted by $(A, C)$ the proposed method, $(\mathbf{B}, \mathbf{D})$ conventional spectral mixture analysis (SMA). 
A quantitative analysis was also conducted in this study for evaluating the performance of the proposed method. One hundred sample points were randomly selected for each study area, Milwaukee County and Fayette County, using a stratified random selection technique (Figure 7). The impervious surface was manually digitized using the software of ERDAS IMAGINE for each selected sampled area, and the abundances of the impervious surface were calculated by dividing the areas of the digitized impervious surface by the total areas of the sampling place. Finally, the estimation accuracy of the abundances of impervious surfaces for both Milwaukee County and Fayette County are included in Table 1. It indicates that the proposed method has achieved a very good performance in mapping the abundance of impervious surfaces for both Milwaukee County and Fayette County. Specifically, in Milwaukee County, the whole study area had an SE of $-2.61 \%$ and MAE of $10.13 \%$. For Fayette County, the SE and MAE of the whole study area was $-4.11 \%$ and $12.48 \%$, respectively. In order to assess the model performance in detail, the whole study area was separated into developed areas and less developed areas based on the definition of developed land from the Environmental Protection Agency [61]. In particular, the developed areas were defined as having fractional impervious surfaces equal or over $30 \%$, and less developed areas were defined as having fractional impervious surfaces smaller than 30\% [61]. Further analysis has shown that much better performance was found in the less developed areas ( $\mathrm{SE}=4.45 \%$ (Milwaukee), 4.01\% (Fayette), and MAE $=9.21 \%$ (Milwaukee), $8.63 \%$ (Fayette) ) than the developed areas (SE $=-5.83 \%$ (Milwaukee), $-7.31 \%$ (Fayette), and MAE $=12.76 \%$ (Milwaukee), 14.17\% (Fayette)). Moreover, a slight overestimation in the less developed areas and underestimation in the developed areas were found for both Milwaukee County and Fayette County.
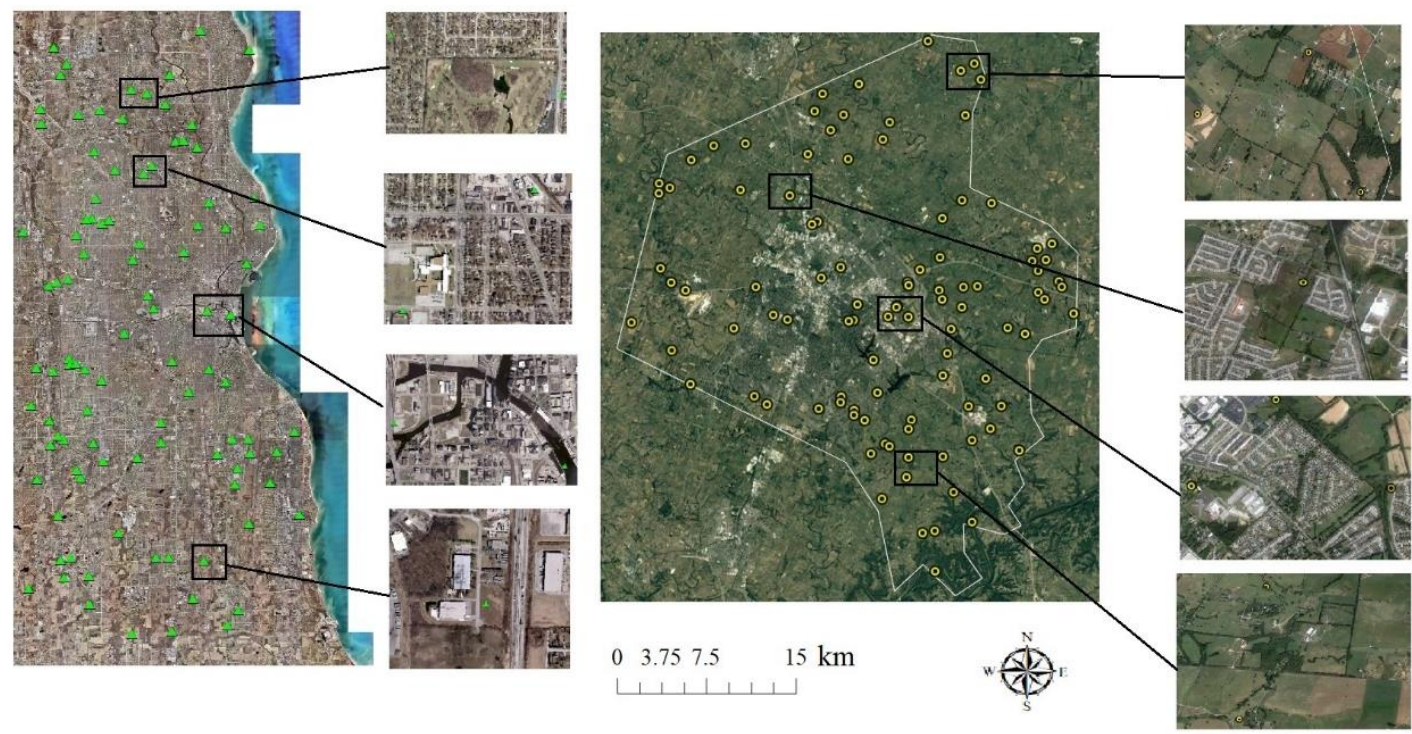

Figure 7. The sample points over the Milwaukee County and Fayette County (background images are the collected fine resolution images used as reference data). 
Table 1. Accuracy assessment of impervious surfaces derived with the proposed method and conventional SMA.

\begin{tabular}{ccccc}
\hline Study Area & Methods & Sub-Area & SE (\%) & MAE (\%) \\
\hline & SMA with spectral indices & Overall $^{1}$ & -2.61 & 10.13 \\
Milwaukee & & Developed areas ${ }^{2}$ & -5.83 & 12.76 \\
County & Conventional & Less developed areas & 4.45 & 9.21 \\
& SMA & Overall & -3.75 & 12.57 \\
& & Developed areas & -7.95 & 13.24 \\
& SMA with spectral indices & Less developed areas & 4.23 & 8.15 \\
\hline & & Overall & -4.11 & 12.48 \\
Fayette & & Developed areas & -7.31 & 14.17 \\
& \multirow{4}{*}{ County } & Less developed areas & 4.01 & 8.63 \\
& SMA & Overall & -6.29 & 13.01 \\
& & Developed areas & -9.17 & 15.54 \\
\end{tabular}

${ }^{1}$ Overall: the whole study area. ${ }^{2}$ Developed areas: fractional impervious surfaces $\geq 30 \% .{ }^{3}$ Less developed areas: fractional impervious surfaces $<30 \%$.

\subsection{Comparative Analysis}

For comparison purposes, the conventional SMA was also implemented, and the performance of the conventional SMA was also included in Figure 6 and Table 1. Figure 6 indicates that similar spatial distribution patterns were achieved for both the proposed method and the conventional SMA. However, compared with the proposed method, an obvious underestimation for the urban area could be detected by the conventional SMA. Quantitative analysis confirms the visual observations. For the whole study area and developed areas, the proposed method has achieved a better performance than the conventional SMA. For instance, for the whole study area, the SE and MAE values of the proposed method are $-2.61 \%$ (Milwaukee), $-4.11 \%$ (Fayette), and 10.13\% (Milwaukee), $12.48 \%$ (Fayette), respectively, much lower than the conventional SMA with an SE of $-3.75 \%$ (Milwaukee), $-6.29 \%$ (Fayette) and MAE of $12.57 \%$ (Milwaukee), 13.01\% (Fayette), respectively. Moreover, in developed areas, the SE and MAE values of the proposed method are $-5.83 \%$ (Milwaukee), $-7.31 \%$ (Fayette) and $12.76 \%$ (Milwaukee), 14.17\% (Fayette), respectively, much lower than the conventional SMA with the SE and MAE of $-7.95 \%$ (Milwaukee), $-9.17 \%$ (Fayette), and $13.24 \%$ (Milwaukee), $15.54 \%$ (Fayette). In summary, the proposed method has achieved much better performance for the whole study area and developed areas, and comparable performance for the less developed areas.

\section{Discussion}

In this study, both SMA and spectral indices were employed to quantify fractional impervious surfaces. The spatial distribution of fractional impervious surfaces in both Milwaukee County and Fayette County are well-reflected. When implementing SMA, one of the most important steps is the endmember selection and extraction. While the traditional statistic methods, such as MNF and PCA, were widely applied [23,46,48,62], the selection and extraction of endmembers are still challenging. Spectral indices seem to be helpful in endmember selection and extraction. Specifically, major land covers can be more accurately identified through spectral indices calculation, and the generated two-dimensional space plots are more straightforward and easily applied in guiding the endmember selection and extraction. Compared with the results derived from the conventional SMA, the performance of the proposed method is much better in the whole study area and developed areas. The improvement in the whole study area is significant (Milwaukee: SE of $-2.61 \%$ vs. $-3.75 \%$ and MAE of $10.13 \%$ vs. $12.57 \%$; Fayette: SE of $-4.11 \%$ vs. $-6.29 \%$ and $12.48 \%$ vs. $13.01 \%$ ). Moreover, a more significant improvement could be found in the developed areas (Milwaukee: SE of $-5.83 \%$ vs. $-7.95 \%$ and MAE of $12.76 \%$ vs. $13.24 \%$; Fayette: SE of $-7.31 \%$ vs. $-9.17 \%$ and MAE of $14.17 \%$ vs. $15.54 \%$ ). While less satisfying performance was achieved in the less developed areas, they are still 
comparable to the conventional SMA. Hence, the use of both SMA and spectral indices produced a more accurate urban impervious surface map than studies only using SMA.

While the proposed method has achieved a satisfactory performance for mapping the abundances of impervious surfaces, there are still some limitations. For instance, there is a slight overestimation in the less developed areas and underestimation in the developed areas due to the variability issue of both endmember class and spectra. In fact, because of the spatial variations of physical and socio-economic forces, endmember spectra are varied from place to place, and a fixed endmember spectrum cannot be enough to represent the complex geographic conditions throughout the whole study area, thereby inevitably generating the estimation error. Moreover, endmember class could also be varied from location to location. If an endmember class is not existing but included in the spectral mixture analysis, the modeled abundances of the non-existing endmember class are usually larger than zero, which may result in large overestimation mistakes. Therefore, further work is still needed for improving the performance of spectral mixture analysis by incorporating both spatial and temporal knowledge into the SMA process. Such knowledge may be helpful in handling the issues of both endmember class and spectra variability and finally addressing the challenging problems of the impervious surface overestimation in the less developed areas and underestimation in the developed areas. Moreover, this study only tested and verified the performance of the proposed method for mapping impervious surfaces in Milwaukee County and Fayette County, so further work is still needed for testing and verifying the model performance in other areas with different natural and human conditions.

\section{Conclusions}

Impervious surface is a very important component of the urbanized area, and several methods have been proposed and applied for quantifying the abundances of impervious surfaces. While the SMA has been broadly considered as one of the most efficient methods, it is still challenging to accurately extract impervious surface. In addition, many spectral indices have also been introduced and applied in mapping urban features, and the effectiveness has also been reported in many studies. In order to improve the impervious surfaces mapping, spectral mixture analysis and spectral indices were integrated together for mapping impervious surfaces in Milwaukee County and Fayette County. This article started at mapping major land covers through calculating spectral indices. Furthermore, four two-dimensional feature spaces were generated to guide the endmember selection and extraction. Finally, a constrained linear SMA was applied to estimate the abundances of impervious surfaces.

Research results suggest some major conclusions. Firstly, the proposed method has achieved a promising performance for quantifying the abundances of impervious surfaces for both Milwaukee County (SE of $-2.61 \%$, MAE of $10.13 \%$ ) and Fayette County (SE of $-4.11 \%$, MAE of $12.48 \%$ ) in the whole study area. Further analysis has also indicated better modeling results in the less developed areas than the developed areas for both Milwaukee County and Fayette County. Secondly, to compare the modeling results of the proposed method with the conventional SMA, both were conducted for mapping the abundances of impervious surfaces for Milwaukee County and Fayette County. Accuracy assessment shows that the estimation accuracy of the proposed method is much better than the conventional SMA with the SE of $-2.61 \%$ (Milwaukee), $-4.11 \%$ (Fayette) (conventional SMA: $-3.75 \%$ (Milwaukee), $-6.29 \%$ (Fayette)) and MAE of $10.13 \%$ (Milwaukee), $12.48 \%$ (Fayette) (conventional SMA: $12.57 \%$ (Milwaukee), $13.01 \%$ (Fayette)). Further analysis has also shown that the estimation accuracy of the proposed method is better than the conventional SMA in the developed areas. Moreover, a comparable performance was achieved by the proposed method and conventional SMA in the less developed areas.

Funding: This research was supported by the University of North Carolina-Greensboro Faculty First Award.

Acknowledgments: I would like to acknowledge the anonymous reviewers for their constructive and valuable suggestions on the earlier drafts of this manuscript.

Conflicts of Interest: The author declares no conflict of interest. 


\section{References}

1. United Nations. World Urbanization Prospects: The 2018 Revision; UN Department of Economic and Social Affairs: New York, NY, USA, 2018.

2. Weng, Q. Remote sensing of impervious surfaces in the urban areas: Requirements, methods, and trends. Remote Sens. Environ. 2012, 117, 34-49. [CrossRef]

3. Wu, C.S.; Murray, A.T. Estimating impervious surface distribution by spectral mixture analysis. Remote Sens. Environ. 2003, 84, 493-505. [CrossRef]

4. Li, W.; Wu, C.; Zang, S. Modeling urban land use conversion of Daqing City, China: A comparative analysis of "top-down" and "bottom-up" approaches. Stoch. Environ. Res. Risk Assess. 2014, 28, 817-828. [CrossRef]

5. Arnold, C.L.; Gibbons, C.J. Impervious surface coverage: The emergence of a key environmental indicator. J. Am. Plan. Assoc. 1996, 62, 243-258. [CrossRef]

6. Goetz, S.J.; Jantz, P.; Jantz, C.A. Connectivity of core habitat in the Northeastern United States: Parks and protected areas in a landscape context. Remote Sens. Environ. 2009, 113, 1421-1429. [CrossRef]

7. Xian, G.; Crane, M. Assessments of urban growth in the Tampa Bay watershed using remote sensing data. Remote Sens. Environ. 2005, 97, 203-215. [CrossRef]

8. $\mathrm{Li}, \mathrm{W} . ; \mathrm{Wu}, \mathrm{C}$. A spatially explicit method to examine the impact of urbanisation on natural ecosystem service values. J. Spat. Sci. 2013, 58, 275-289. [CrossRef]

9. Bauer, M.E.; Heinert, J.J.; Doyle, J.K.; Yuan, F. Impervious surface mapping and change monitoring using Landsat remote sensing. In ASPRS Annual Conference Proceedings; American Society for Photogrammetry and Remote Sensing: Bethesda, MD, USA, 2004; Volume 10.

10. Foody, G.M.; Cox, D.P. Sub-pixel land-cover composition estimation using a linear mixture model and fuzzy membership functions. Int. J. Remote Sens. 1994, 15, 619-631. [CrossRef]

11. Ridd, M.K. Exploring a VIS (vegetation-impervious surface-soil) model for urban ecosystem analysis through remote sensing-comparative anatomy for cities. Int. J. Remote Sens. 1995, 16, 2165-2185. [CrossRef]

12. Wu, C.S. Normalized spectral mixture analysis for monitoring urban composition using ETM plus imagery. Remote Sens. Environ. 2004, 93, 480-492. [CrossRef]

13. Lee, S.; Lathrop, R.G. Subpixel analysis of Landsat ETM/sup+/using self-organizing map (SOM) neural networks for urban land cover characterization. IEEE Trans. Geosci. Remote Sens. 2006, 44, 1642-1654.

14. Lu, D.; Weng, Q. Extraction of urban impervious surfaces from an IKONOS image. Int. J. Remote Sens. 2009, 30, 1297-1311. [CrossRef]

15. Yang, L.M.; Huang, C.Q.; Homer, C.G.; Wylie, B.K.; Coan, M.J. An approach for mapping large-area impervious surfaces: Synergistic use of Landsat-7 ETM+ and high spatial resolution imagery. Can. J. Remote Sens. 2003, 29, 230-240. [CrossRef]

16. Mohapatra, R.P.; Wu, C. High resolution impervious surface estimation. Photogramm. Eng. Remote Sens. 2010, 76, 1329-1341. [CrossRef]

17. Yang, L.M.; Xian, G.; Klaver, J.M.; Deal, B. Urban land-cover change detection through sub-pixel imperviousness mapping using remotely sensed data. Photogramm. Eng. Remote Sens. 2003, 69, 1003-1010. [CrossRef]

18. Hu, X.; Weng, Q. Estimating impervious surfaces from medium spatial resolution imagery using the self-organizing map and multi-layer perceptron neural networks. Remote Sens. Environ. 2009, 113, 2089-2102. [CrossRef]

19. Yuan, F.; Bauer, M.E. Comparison of impervious surface area and normalized difference vegetation index as indicators of surface urban heat island effects in Landsat imagery. Remote Sens. Environ. 2007, 106, 375-386. [CrossRef]

20. Yuan, F.; $\mathrm{Wu}, \mathrm{C}$;; Bauer, M.E. Comparison of spectral analysis techniques for impervious surface estimation using Landsat imagery. Photogramm. Eng. Remote Sens. 2008, 74, 1045-1055. [CrossRef]

21. Lu, D.; Moran, E.; Hetrick, S. Detection of impervious surface change with multitemporal Landsat images in an urban-rural frontier. ISPRS J. Photogramm. 2011, 66, 298-306. [CrossRef]

22. Plaza, A.; Martinez, P.; Perez, R.; Plaza, J. A quantitative and comparative analysis of endmember extraction algorithms from hyperspectral data. IEEE Trans. Geosci. Remote Sens. 2004, 42, 650-663. [CrossRef]

23. Somers, B.; Asner, G.P.; Tits, L.; Coppin, P. Endmember variability in spectral mixture analysis: A review. Remote Sens. Environ. 2011, 115, 1603-1616. [CrossRef] 
24. Weng, Q.; Hu, X.; Lu, D. Extracting impervious surfaces from medium spatial resolution multispectral and hyperspectral imagery: A comparison. Int. J. Remote Sens. 2008, 29, 3209-3232. [CrossRef]

25. Wu, C. Quantifying high-resolution impervious surfaces using spectral mixture analysis. Int. J. Remote Sens. 2009, 30, 2915-2932. [CrossRef]

26. Li, W.; Wu, C.; Choi, W. Predicting future urban impervious surface distribution using cellular automata and regression analysis. Earth Sci. Inform. 2018, 11, 19-29. [CrossRef]

27. Bedini, E. Mapping lithology of the Sarfartoq carbonatite complex, southern West Greenland, using HyMap imaging spectrometer data. Remote Sens. Environ. 2009, 113, 1208-1219. [CrossRef]

28. Johnson, P.E.; Smith, M.O.; Taylorgeorge, S.; Adams, J.B. A semiempirical method for analysis of the reflectance spectra of binary mineral mixtures. J. Geogr. Res. 1993, 88, 3557-3561. [CrossRef]

29. Ramsey, M.S.; Christensen, P.R. Mineral abundance determination: Quantitative deconvolution of thermal emission spectra. J. Geophys. Res. Solid Earth 1998, 103, 577-596. [CrossRef]

30. Jia, G.J.; Burke, I.C.; Goetz, A.F.H.; Kaufmann, M.R.; Kindel, B.C. Assessing spatial patterns of forest fuel using AVIRIS data. Remote Sens. Environ. 2006, 102, 318-327. [CrossRef]

31. Eckmann, T.C.; Still, C.J.; Roberts, D.A.; Michaelsen, J.C. Variations in subpixel fire properties with season and land cover in southern Africa. Earth Interact. 2010, 14, 1-29. [CrossRef]

32. Small, C. Estimation of urban vegetation abundance by spectral mixture analysis. Int. J. Remote Sens. 2001, 22, 1305-1334. [CrossRef]

33. Song, C.H. Spectral mixture analysis for subpixel vegetation fractions in the urban environment: How to incorporate endmember variability? Remote Sens. Environ. 2005, 95, 248-263. [CrossRef]

34. Liu, T.; Yang, X. Mapping vegetation in an urban area with stratified classification and multiple endmember spectral mixture analysis. Remote Sens. Environ. 2013, 133, 251-264. [CrossRef]

35. Small, C.; Lu, J.W.T. Estimation and vicarious validation of urban vegetation abundance by spectral mixture analysis. Remote Sens. Environ. 2006, 100, 441-456. [CrossRef]

36. Weng, Q.; Hu, X. Medium spatial resolution satellite imagery for estimating and mapping urban impervious surfaces using LSMA and ANN. IEEE Trans. Geosci. Remote Sens. 2008, 46, 2397-2406. [CrossRef]

37. Lu, D.; Weng, Q. Use of impervious surface in urban land-use classification. Remote Sens. Environ. 2006, 102, 146-160. [CrossRef]

38. Madhavan, B.B.; Kubo, S.; Kurisaki, N.; Sivakumar, T. Appraising the anatomy and spatial growth of the Bangkok Metropolitan area using a vegetation-impervious-soil model through remote sensing. Int. J. Remote Sens. 2001, 22, 789-806. [CrossRef]

39. Phinn, S.; Stanford, M.; Scarth, P.; Murray, A.T.; Shyy, P.T. Monitoring the composition of urban environments based on the vegetation-impervious surface-soil (VIS) model by subpixel analysis techniques. Int. J. Remote Sens. 2002, 23, 4131-4153. [CrossRef]

40. Powell, R.L.; Roberts, D.A.; Dennison, P.E.; Hess, L.L. Sub-pixel mapping of urban land cover using multiple endmember spectral mixture analysis: Manaus, Brazil. Remote Sens. Environ. 2007, 106, 253-267. [CrossRef]

41. Ward, D.; Phinn, S.R.; Murray, A.T. Monitoring growth in rapidly urbanizing areas using remotely sensed data. Prof. Geogr. 2000, 52, 371-386. [CrossRef]

42. Weng, Q.; Hu, X.; Liu, H. Estimating impervious surfaces using linear spectral mixture analysis with multitemporal ASTER images. Int. J. Remote Sens. 2009, 30, 4807-4830. [CrossRef]

43. Hu, X.; Weng, Q. Estimating impervious surfaces from medium spatial resolution imagery: A comparison between fuzzy classification and LSMA. Int. J. Remote Sens. 2011, 32, 5645-5663. [CrossRef]

44. Michishita, R.; Jiang, Z.; Xu, B. Monitoring two decades of urbanization in the Poyang Lake area, China through spectral unmixing. Remote Sens. Environ. 2012, 117, 3-18. [CrossRef]

45. Zhang, J.; He, C.; Zhou, Y.; Zhu, S.; Shuai, G. Prior-knowledge-based spectral mixture analysis for impervious surface mapping. Int. J. Appl. Earth Obs. Geoinf. 2014, 28, 201-210. [CrossRef]

46. Zhang, J.K.; Rivard, B.; Sanchez-Azofeifa, A. Derivative spectral unmixing of hyperspectral data applied to mixtures of lichen and rock. IEEE Trans. Geosci. Remote Sens. 2004, 42, 1934-1940. [CrossRef]

47. Li, M.; Zang, S.; Wu, C.; Deng, Y. Segmentation-based and rule-based spectral mixture analysis for estimating urban imperviousness. Adv. Space Res. 2015, 55, 1307-1315. [CrossRef]

48. Chang, C.I.; Ji, B.H. Weighted abundance-constrained linear spectral mixture analysis. IEEE Trans. Geosci. Remote Sens. 2006, 44, 378-388. [CrossRef] 
49. Deng, C.; Wu, C. A spatially adaptive spectral mixture analysis for mapping subpixel urban impervious surface distribution. Remote Sens. Environ. 2013, 133, 62-70. [CrossRef]

50. Roberts, D.A.; Gardner, M.; Church, R.; Ustin, S.; Scheer, G.; Green, R.O. Mapping chaparral in the Santa Monica mountains using multiple endmember spectral mixture models. Remote Sens. Environ. 1998, 65, 267-279. [CrossRef]

51. Li, W.; Wu, C. Phenology-based temporal mixture analysis for estimating large-scale impervious surface distributions. Int. J. Remote Sens. 2014, 35, 779-795. [CrossRef]

52. Li, W. Examining the importance of endmember class and spectra variability in unmixing analysis for mapping urban impervious surfaces. Adv. Space Res. 2017, 60, 2389-2401. [CrossRef]

53. Li, W.; Wu, C. A geostatistical temporal mixture analysis approach to address endmember variability for estimating regional impervious surface distributions. GISci. Remote Sens. 2016, 53, 102-121. [CrossRef]

54. Li, W.; Wu, C. A geographic information-assisted temporal mixture analysis for addressing the issue of endmember class and endmember spectra variability. Sensors 2017, 17, 624. [CrossRef] [PubMed]

55. Zha, Y.; Gao, J.; Ni, S. Use of normalized difference built-up index in automatically mapping urban areas from TM imagery. Int. J. Remote Sens. 2003, 24, 583-594. [CrossRef]

56. Deng, C.; Wu, C. BCI: A biophysical composition index for remote sensing of urban environments. Remote Sens. Environ. 2012, 127, 247-259. [CrossRef]

57. Deng, Y.; Wu, C.; Li, M.; Chen, R. RNDSI: A ratio normalized difference soil index for remote sensing of urban/suburban environments. Int. J. Appl. Earth Obs. Geoinf. 2015, 39, 40-48. [CrossRef]

58. Rouse, J.W., Jr.; Haas, R.; Schell, J.; Deering, D. Monitoring Vegetation Systems in the Great Plains with ERTS; Technical Report No. PAPER-A20; NASA: College Station, TX, USA, 1974.

59. Shih, H.-C.; Stow, D.A.; Tsai, Y.-M.; Roberts, D.A. Estimating the starting time and identifying the type of urbanization based on dense time series of landsat-derived Vegetation-Impervious-Soil (VIS) maps-A case study of North Taiwan from 1990 to 2015. Int. J. Appl. Earth Obs. Geoinf. 2020, 85, 101987. [CrossRef]

60. Lippitt, C.L.; Stow, D.A.; Roberts, D.A.; Coulter, L.L. Multidate MESMA for monitoring vegetation growth forms in southern California shrublands. Int. J. Remote Sens. 2018, 39, 655-683. [CrossRef]

61. EPA. Definitions of Land Cover Categories for Exhibits 4-1, 4-2, and 4-3. Available online: https://cfpub.epa. gov/si/si_public_file_download.cfm?p_download_id=494738 (accessed on 10 August 2019).

62. Asner, G.P.; Lobell, D.B. A biogeophysical approach for automated SWIR unmixing of soils and vegetation. Remote Sens. Environ. 2000, 74, 99-112. [CrossRef] 